\title{
Comunicaciones Satelitales al servicio de la Industria Marítima.
}

\section{Ricaurte Arosemena M. . $^{*}$}

${ }^{1}$ Oficial Radio Electrónico de Primera Clase; Profesor de la Facultad de Ingeniería y Tecnología, Universidad Católica Santa María la Antigua.

*Autor para correspondencia. E-mail: rarosemena@usma.com.pa

Recibido: 18 de noviembre de 2019

Aceptado: 20 de diciembre de 2019

\section{Resumen}

Las Comunicaciones Satelitales es un activo importante para la industria Marítima, el cual permite dar seguimiento a los bienes que transporta, guardar la vida humana en los océanos, y mantener la conexión de la tripulación con sus seres queridos. En los últimos 25 años se ha crecimiento de la demanda de las comunicaciones Satelitales a pesar de la red de fibra óptica que cube el planeta, sin olvidar que hay muchos lugares remotos, aislado o de difícil acceso el cual las telecomunicaciones terrestres no están disponibles. En este artículo se describirá las soluciones en comunicaciones marítimas satelitales, descripción y aplicaciones, se verá el futuro de las comunicaciones Satelitales marítimas para los próximos 20 años.

Palabras clave: Inmarsat, Iridium, Satelital, GMDSS, SSAS, LRIT, GPS, banda L, Servicios satelitales marítimos MSS, red de seguridad

\begin{abstract}
Basic The Satellite Communications is an important asset for the Maritime industry, which allows us to track the goods it transports, guarantee human life in the oceans, and maintain the connection of the crew with their loved ones. In the last 25 years there has been growth in the demand for satellite communications despite the fiber optic network that covers the planet, without forgetting that there are many remote places, isolated or difficult to access which terrestrial telecommunication is not available. In this article we will describe the solutions in maritime satellite communications, description and applications, we will see the future of maritime satellite communications for the next 20 years.

Keywords: Inmarsat, Iridium, Satellite, GMDSS, SSAS, LRIT, GPS, L-band, MSS maritime satellite services, safety net.
\end{abstract}




\section{Introducción}

Las Comunicaciones Marítimas tuvo sus inicios del siglos de existir, por medio de señalización por banderas, señales de luz. Con la invención de la radio por Marconi a finales del siglo XIX y el mismo creo una empresa que alquilaba estaciones de radio a los buques mercante junto con los operadores de radio. Un evento que marco mucho la seguridad en mar fue el naufragio del Titanic en cual la estación de radio y sus operadores jugaron un papel importante en el rescate de los sobrevivientes.

Hoy en días hay comunicaciones por medio de ondas de radio VHF y MF/HF en esta ocasión se escriba sobre las comunicaciones satelitales Marítimas. El 16 de Julio de 1976 se fundo Inmarsat como empresa sin fines de lucro formada por Estados miembros, inicio alquilando 2 satélites a Comsat, los cuales no estaban dispuestos a invertir en mejoras a la red de INMARSAT, actualmente es el cual la primera empresa en poner un sistema de comunicaciones a los barcos, siendo hoy en días la principal empresa proveedores de servicios de GMDSS.

\section{INMARSAT}

En abril de 1999 INMARSAT fue sucedido por la Organización Internacional de Satélites Móviles (IMSO) como organismo regulador intergubernamental para las comunicaciones por satélite, mientras que la unidad operacional de INMARSAT fue separada y se convirtió en la empresa británica Inmarsat Ltd. La IMSO e Inmarsat Ltd. firmaron un acuerdo que imponía obligaciones de seguridad pública a la nueva compañía. Inmarsat fue la primera organización internacional de satélites que fue privatizada.

En 2005, Apax Partners y Permira compraron acciones de la compañía. La compañía también se cotizó por primera vez en la Bolsa de Londres en ese año. En marzo de 2008, se reveló que el fondo de cobertura estadounidense Harbinger Capital poseía el 28\% de la compañía. En 2009, Inmarsat completó la adquisición del proveedor de comunicaciones satelitales Stratos Global Corporation (Stratos) y adquirió una participación del 19 por ciento en SkyWave Mobile Communications Inc., un proveedor de servicios de red Inmarsat D + / IsatM2M que a su vez adquirió el negocio de GlobalWave de TransCore. Inmarsat ganó el premio MacRobert 2010 por su servicio de red de área global de banda ancha (BGAN).

\section{Segmento espacial.}

Inmarsat durante su trayectoria ha tenido en órbita a 16 satélites actualmente tiene actualmente están operativas las generaciones I-4 e I-5 son satélites geoestacionarios en orbital ecuatorial a una distancia de 36,780 mil kilómetros de la tierra.

Los satélites Inmarsat-4 (I-4) estableció la primera red móvil global 3G del mundo. Ofrecen nuestros servicios de banda ancha de banda L: BGAN, FleetBroadband y SwiftBroadband; IoT de máquina a máquina (M2M); y servicios de voz. Desde 2018 también han impulsado nuestros servicios heredados de seguridad marítima y de aviación, migrados de los tres satélites I-3 que aún están en servicio.

Los tres primeros satélites Inmarsat-4 (I-4) se lanzaron a la órbita geoestacionaria desde 2005-8. 
Cada satélite puede generar hasta 19 haces anchos y más de 200 haces puntuales estrechos. En 2010, el diseño y la capacidad del satélite I-4.

En julio de 2013, lanzamos Alphasat para complementar la flota de I-4, brindando cobertura en Europa, Medio Oriente y África y brindando nuevas capacidades que incluyen un $50 \%$ más de espectro de banda L accesible y casi un 20\% más de canales de comunicación móvil.

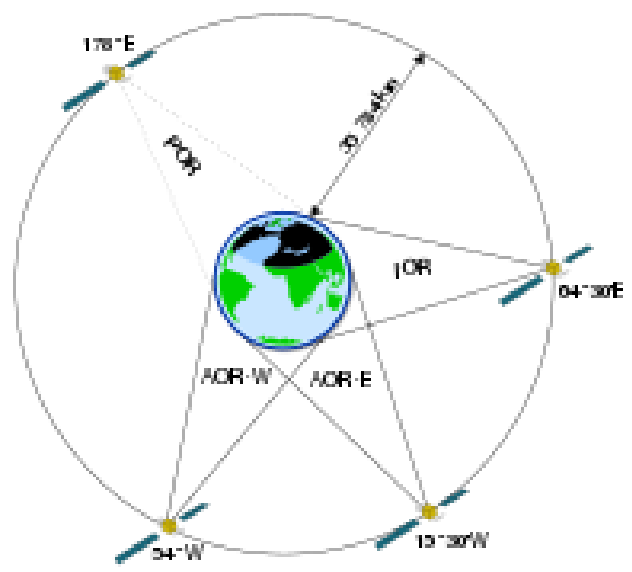

Imagen 1; Orbita de satélites Inmarsat.

Se espera que la flota de Inmarsat-4 respalde nuestros servicios de banda L hasta bien entrados los años 2020.

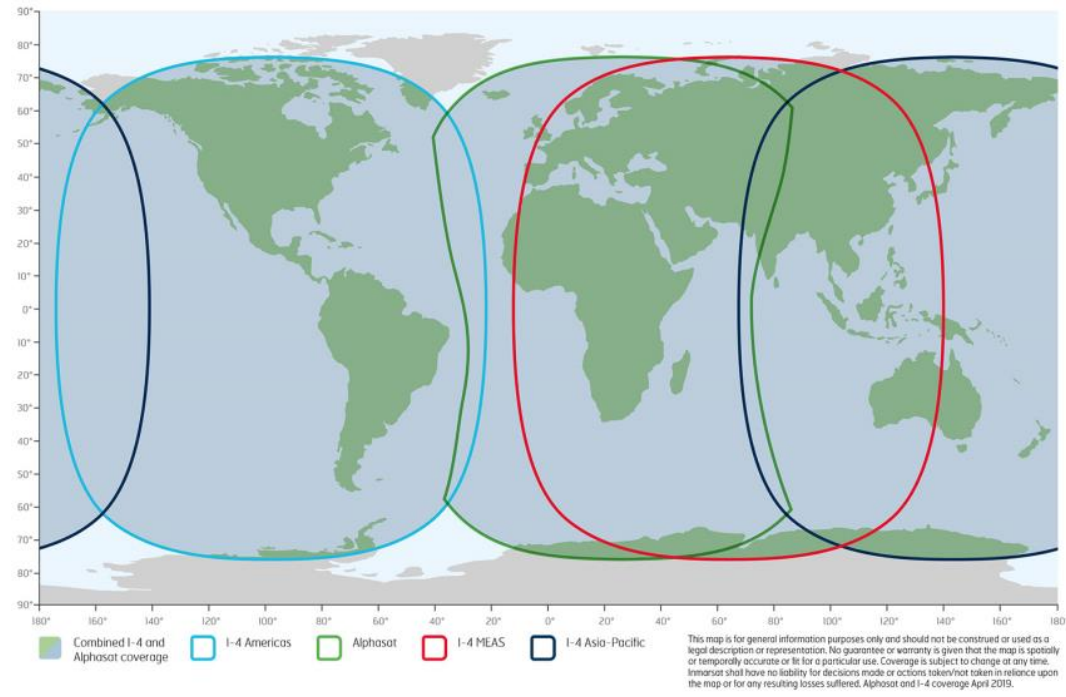

Imagen 2: Cobertura de los Satélites de Inmarsat.

Los satélites Inmarsat I-5 de banda Ka de quinta generación de Inmarsat impulsan a Global Xpress (GX), la primera y única red de banda ancha móvil de alta velocidad que se extiende por todo el mundo. El galardonado servicio líder en el mercado está transformando industrias enteras al ofrecer soluciones de conectividad altamente confiables y totalmente escalables. 
Los satélites Inmarsat-5 (I-5) fueron construidos por el fabricante estadounidense Boeing, basándose en su potente plataforma 702HP. Los primeros tres satélites I-5 se lanzaron entre diciembre de 2013 y agosto de 2015, y el servicio comercial global comenzó en diciembre de 2015. Se lanzó un cuarto satélite GX en mayo de 2017 para proporcionar capacidad adicional.

(Latamsatelital 2019) En Julio del 2019 Inmarsat anuncio un hecho histórico inicio un programa de colocar 2 satélites de global express con orbita elíptica para brindar cobertura en el árctico para el año 2022. En una asociación con Space Norway y su filial Space Norway HEOSAT, se colocarán dos cargas útiles de satélites, GX10A y 10B, en órbitas altamente elípticas (HEO), asegurando una cobertura continua por encima de $65^{\circ}$ Norte.

La nueva iniciativa de Inmarsat es cumplir con la demanda de banda ancha móvil de alta velocidad, no solamente en la región del Ártico, sino que en todo el mundo. Y equipara la balanza de cobertura frente a la capacidad existente del operador satelital Iridium en cubrir brindar servicio de banda ancha móvil de alta velocidad en las zonas polares.

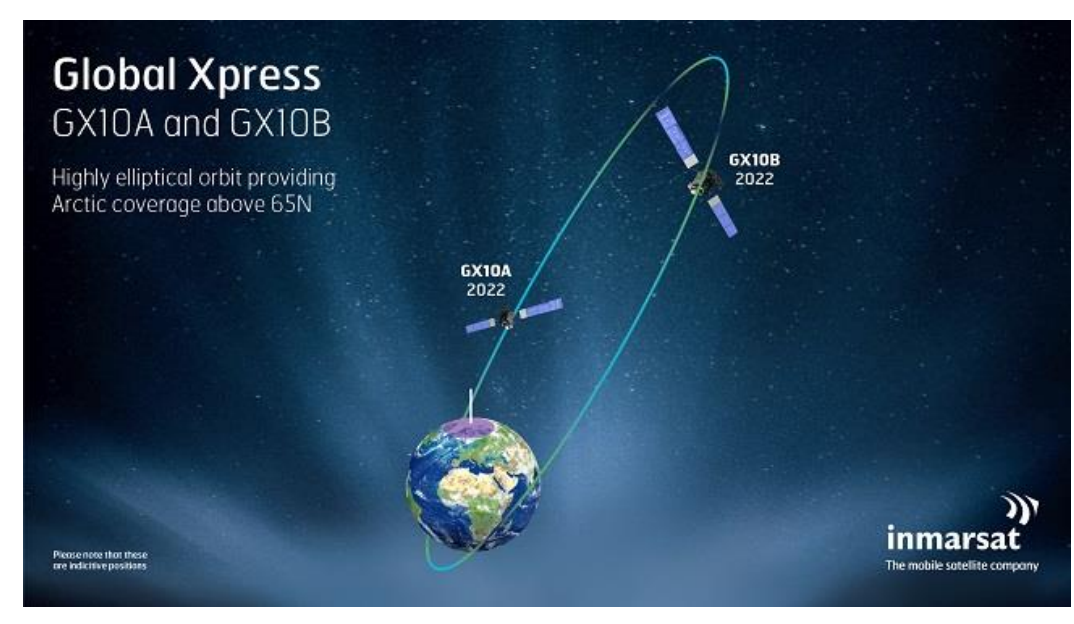

Imagen 4: Cobertura en el ártico por los satélites GlobalExpress

\section{Soluciones de Comunicación satelital de Inmarsat.}

Se presentará una breve descripción de sistemas ya fuera de servicio solo para tener una idea de la evolución de las comunicaciones satelitales marítimas. Posterior la descripción de sistemas actuales en servicio.

Inmarsat-A fue el primer servicio que se introdujo y se comercializó en 1982. Un sistema analógico, que proporciona un teléfono bidireccional de marcación directa, fax, télex y correo electrónico y comunicaciones de datos a velocidades de hasta 9.6 kilobits por segundo (kbit / s). Actualmente descontinuado y los satélites fuera de servicio.

Inmarsat-B se introdujo en 1994 y utilizo tecnología digital para proporcionar calidad Comunicaciones por teléfono, fax, télex, correo electrónico y datos de calidad a hasta 64Kbs, lo que lo hacía adecuado para usuarios con alta demanda de datos, como empresas petroleras y sismológicas, que necesitan intercambiar grandes cantidades de datos sobre una base regular. Actualmente fuera descontinuado. 
El Inmarsat E fue el sistema de alerta de socorro Inmarsat-E ha estado disponible a nivel mundial desde 1997, equipo combinaba las capacidades de determinación de posición del GPS con el satélite geoestacionario. Tecnología del sistema de comunicaciones Inmarsat. Cumple plenamente con el GMDSS. regulaciones, proporciona una cobertura confiable entre latitudes 80o Norte y 80o Sur. Actualmente fuera descontinuado. Actualmente fuera descontinuado.

Inmarsat-M se introdujo en 1993 para complementar el sistema existente Inmarsat-A, al proporcionar comunicaciones globales de teléfono / fax y datos en un MES baja velocidad que era económico y de tamaño compacto.

Actualmente fuera descontinuado.

Inmarsat mini-M se lanzó en enero de 1997 y ofrece los mismos servicios que Inmarsat-M, pero en una unidad más pequeña, más ligera y compacta (de un maletín ejecutivo). La terminal se puedo hacer más pequeño porque operaba solo en la cobertura de haz puntual de los últimos satélites Inmarsat-3, con baterías internas. Actualmente fuera descontinuado.

El Inmarsat Fleet (F77) se lanzó en noviembre de 2001. Ofreció un alto rendimiento de servicio de comunicaciones de alta velocidad de costa a barco y de barco, F77 presento un nuevo servicio de RDSI móvil y Paquetes de datos móviles (MPDS) voz, fax y datos a velocidades de hasta $64 \mathrm{kbit} / \mathrm{s}$. Inmarsat Fleet F77 está equipado para satisfacer las Últimos requisitos de seguridad y socorro del GMDSS. Ofrece datos más eficientes basados en datos.

Comunicaciones para aplicaciones tales como gestión técnica y registro de personal, acceso a una intranet de la oficina central, y la obtención de actualizaciones de información meteorológica video y gráfica. Actualmente fuera de servicio (Handbook, 2004) pagina 13-14.

\section{Sistemas Actuales que cuenta Inmarsat.}

Inmarsat-C se introdujo en 1991 para complementar a Inmarsat-A al proporcionar un bajo costo global una red de comunicaciones de datos de dos vías que utiliza una pequeña terminal, aunque lo hace especialmente adecuado para embarcaciones más pequeñas, como yates, embarcaciones de pesca o embarcaciones de suministro fue ampliamente utilizado en buques mercantes para cumplir con el requerimiento de GMDSS (Global Maritime Distress Safety System). El sistema Inmarsat-C no proporciona comunicaciones de voz, pero es un medio para envío de mensajes de texto, datos, fax y correo electrónico desde y hacia los suscriptores en tierra utilizando una técnica de "Store \& Fordward" almacenamiento y reenvío. Actualmente es una plataforma en servicio después de 28 años, por ser muy práctica, cumple con el requerimiento a bordo de GMDSS. Tecnológicamente a evolucionado en la electrónica, incluyendo conexiones de ethernet para interconectarse a PC por medio de la red abordo. 


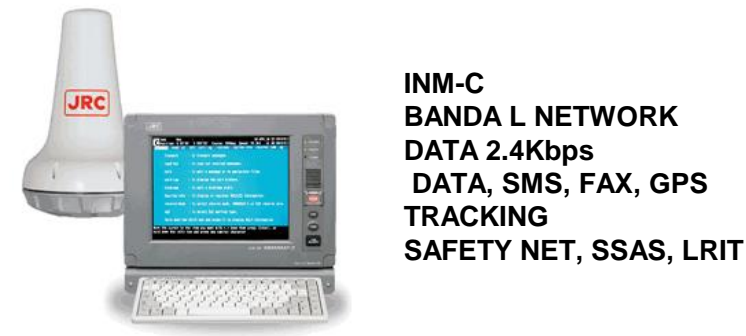

Figura 5: Terminal JRC JUE-87, Inmarsat-C.

(InmarsatInmarsat FleetBroadband FBB 250/500 es el primer servicio de comunicaciones marítimas que ofrece soluciones rentables en la provisión simultánea de datos y voz en banda ancha, globalmente, y a través de una antena compacta. Este servicio de la última generación de Inmarsat lleva a su buque a la era del Protocolo de Internet (IP). Basado en los estándares 3G, FleetBroadband ofrece un acceso constante y simultáneo a servicios de voz y datos a alta velocidad - a nivel global. Puede enviar y recibir correo electrónico con archivos adjuntos de gran tamaño, ejecutar cómodamente complejas aplicaciones de datos y hacer llamadas telefónicas al mismo tiempo.

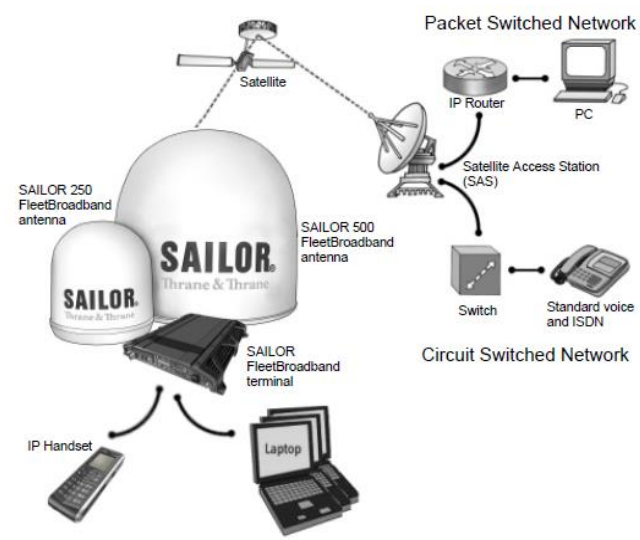

Figura 6: Inmarsat FleetBroadBand.

Prestaciones:

- Fleet Broadband FBB 250/500

- Inmarsat-4 actual

- Inmarsat-6 futuro

- MSI, SafetyNet II, RescueNET y

Distres Alert

- SAR coordinación

- Voz

- SMS

- Ethernet

- Data hasta $1 \mathrm{Mbs}$

- ISDN

- IP Standard

- IP Streaming 


\section{Inmarsat Fleet One.}

Inmarsat 2014) Fleet One fue originalmente diseñado para cumplir con los requisitos de la industria pesquera con operaciones próximas a la costa, hoy cuenta con mucha aceptación entre las embarcaciones de placer, de trabajo y mercantes que operan cerca de las costa o navegación costera. Ver figura 7 de la cobertura del FleetOne. Cuenta con datos y voz simultáneamente a través de un Antena. Para los tripulantes, brinda el acceso a llamadas de bajo costo y correo electrónico los mantiene en contacto con el hogar y aumenta la moral. Con costo bajo de operación.

Sus prestaciones son:

- Data hasta 100Kbps

- Voz 4Kbps

- SMS

- GPS

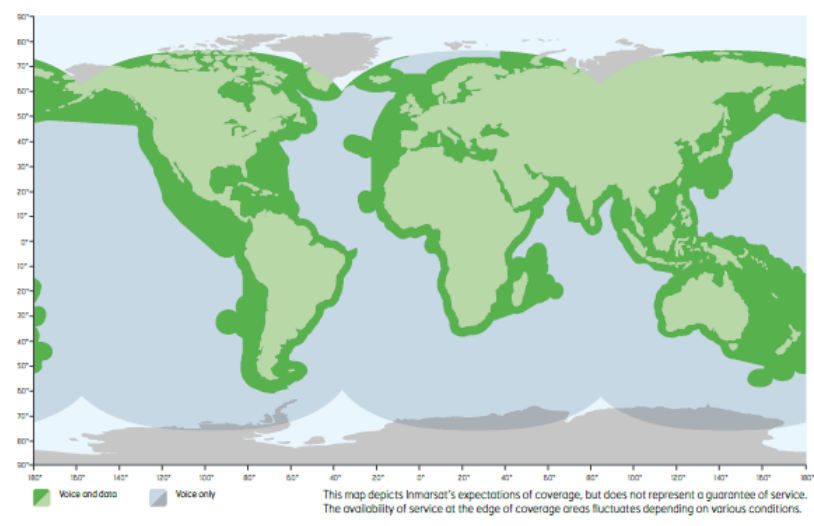

Figura 7: Cobertura del Fleet One.

(Inmarsat/maritime-safety, 2018) En mayo de 2018, el servicio de seguridad satelital de próxima generación provisto por Fleet Broadband y Fleet One, recibió el reconocimiento formal de GMDSS por parte de la OMI, marcando el avance más significativo en seguridad marítima para una generación.

Los servicios SafetyNET, SafetyNET II y RescueNET tienen un papel crucial en la mejora de la seguridad de la vida en el mar transmitiendo información de seguridad marítima y mensajes de información relacionados con la búsqueda y el rescate a las terminales Inmarsat C, Mini C y Fleet Safety.

\section{Inmarsat Global Express.}

La red Global Xpress de Inmarsat es la primera solución en ofrecer banda ancha de extremo a extremo disponible en gran parte del planeta con un único servicio de red de un solo operador de red. Es el servicio VSAT de banda Ka es más avanzado para tierra, mar y aire y ofrece mayor velocidad y comunicaciones más seguras que las soluciones de conectividad satelital global previas.

La empresa Inmarsat está invirtiendo US \$1.6 mil millones en infraestructura terrestre y espacial. La 
nueva constelación "Inmarsat-5" El primer satélite cubre Europa, Medio Oriente, África y Asia, el segundo cubre la región del Océano Atlántico y América, y el tercer satélite cubre la región del Océano Pacífico.

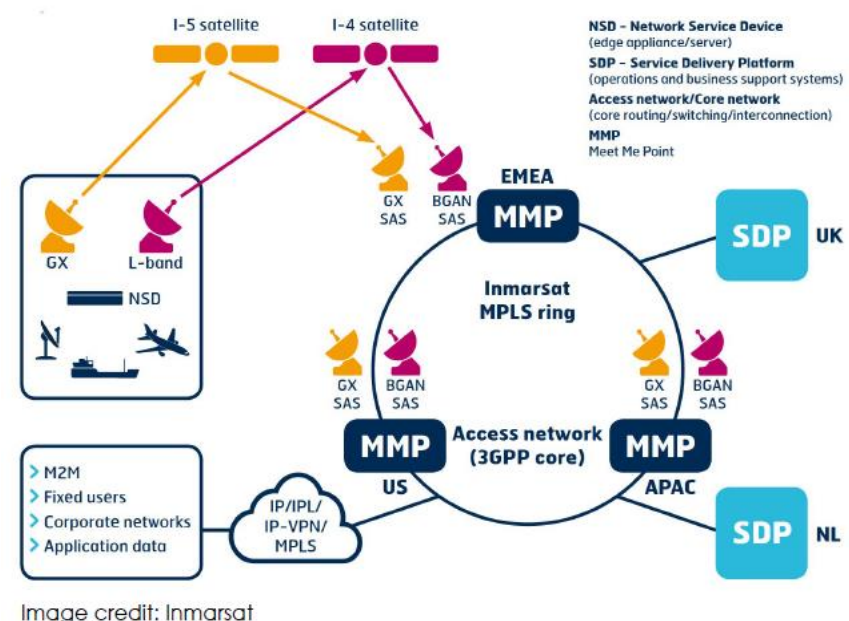

Figura 8: Arquitectura de red de Global Express.

One Touch Commissioning (OTC) es un diseño de sistema único para la capacidad GX y común a todos los terminales GX. Es un concepto por el cual el núcleo módulo, BUC / transceptor y posicionador de terminal (o mecanismo de puntería asistida para terminales manuales) están estrechamente integrados a través de la implementación de open protocolos de comunicación estándar.

Para el usuario final, significa que pueden poner en servicio una terminal GX en la red con poca o ninguna experiencia previa de VSAT y sin tener que contactar a una red centro de operaciones, poner en marcha es especialmente fácil con terminales transportables que incluyen asistentes de alineamiento robustos o sistemas de adquisición automática (Skywave technologies, 2015)

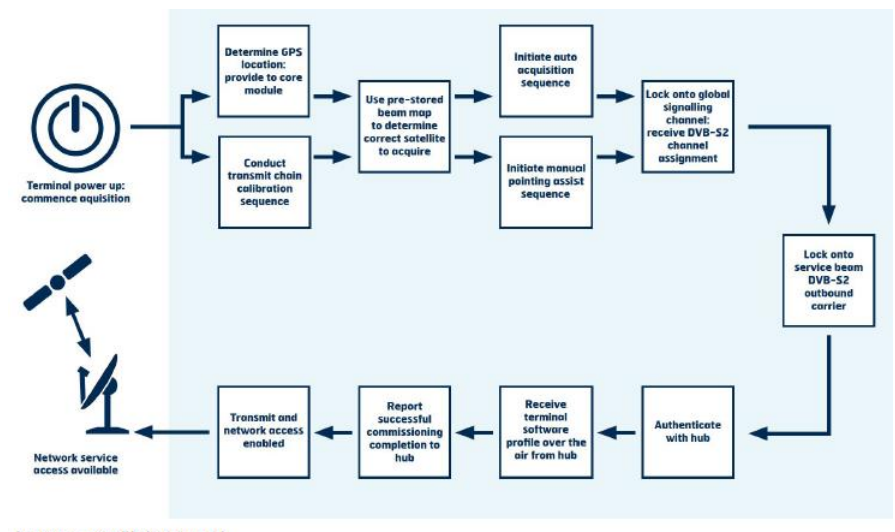

Image credit: Inmarsat

Figura 9: Comisiona miento en un toque (OTC) 


\section{IRIDIUM}

(Satelite, 2016) Reseña histórica: La primera constelación de Iridium estuvo operativa el 1 de noviembre de 1998 orbitales a una altura de alrededor de 780 kilómetros de la Tierra dando cobertura global de telefonía satelital de voz y datos. La constelación Iridium trabaja en Banda L y las comunicaciones entre satélites se realizan en Banda Ka. y quebró financieramente el 13 de agosto de 1999. Esta quiebra fue debida en gran parte al elevado costo de los terminales móviles, Los precios de los teléfonos móviles terrestres, considerablemente más baratos, y la aparición de la telefonía móvil celular GSM.

Otro hecho que contribuyó a la quiebra de Iridium fue su incapacidad para proveer servicios de datos de alta velocidad, puesto que la constelación de satélites fue diseñada esencialmente para comunicaciones de voz. Actualmente Iridium ofrece comunicaciones de datos de $2,4 \mathrm{kbit} / \mathrm{s}$ nativos y un sistema de conexión a internet que emula $10 \mathrm{kbit} / \mathrm{s}$, lo que limita las posibles aplicaciones, por lo que se lo usa, sobre todo, para el envío y recepción de correos electrónicos en formato de texto.

Actualmente las soluciones de Iridium, que fue comprada y relanzada por nuevos socios, se utilizan activamente en mercados verticales como los petroleros, mineros, ecoturismo y militar. Después de los sucesos del 11 de septiembre de 2001 los organismos de seguridad estadounidenses utilizan soluciones Iridium como su sistema preferido de telecomunicaciones satelitales móviles de voz.

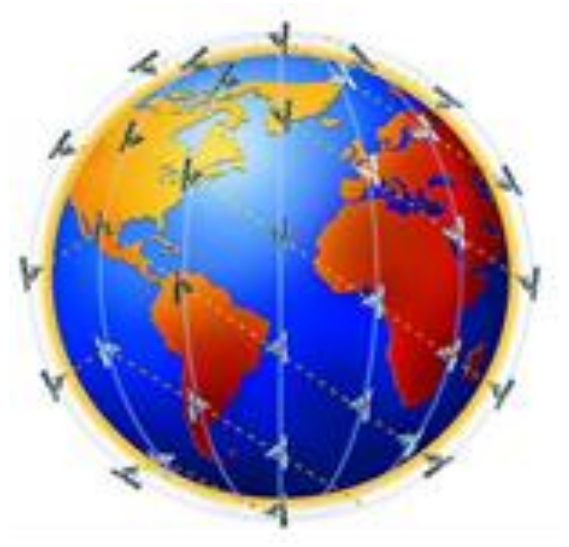

Figura 10. Constelación de Satelites Iridium

\section{Constelación de la próxima generación}

Iridium puso en operación la nueva generación de satélites, Iridium NEXT, una red mundial de segunda generación de satélites de telecomunicaciones, compuesta por 66 satélites, con seis repuestos en órbita y nueve repuestos en tierra. Estos satélites incorporarán características tales como la transmisión de datos que no se enfatizaron en el diseño original. 
Tabla 1: comparación de Red

\begin{tabular}{|c|c|}
\hline Red Actual & Red NEXT \\
\hline $\begin{array}{l}\text { - Arquitectura } \\
\text { interconectada } \\
\text { - } 66 \text { satélites de baja } \\
\text { órbita terrestre } \\
\text { - Múltiples repuestos en } \\
\text { órbita } \\
\text { - } 2 \text { millones de capacidad } \\
\text { de abonado } \\
\text { - Velocidades de datos } \\
\text { de banda L de hasta } 128 \\
\text { kbps Cobertura } \\
\text { - } \\
\text { verdaderamente global } \\
\text { - Alta calidad de voz } \\
\text { latencia fiabilidad y baja }\end{array}$ & $\begin{array}{l}\text { - Arquitectura } \\
\text { interconectada } \\
\text { - } 66 \text { satélites de baja órbita } \\
\text { terrestre } \\
\text { - } 6 \text { repuestos en órbita, } 9 \\
\text { repuestos en el suelo } \\
\text { - } 3 \text { millones de capacidad de } \\
\text { abonado } \\
\text { - Velocidades de datos en } \\
\text { banda L de hasta } 1,5 \text { Mbps } \\
\text { - Servicio de banda Ka de } \\
\text { alta velocidad de hasta } 8 \\
\text { Mbps } \\
\text { - Cobertura verdaderamente } \\
\text { global } \\
\text { - Calidad de voz mejorada } \\
\text { - Alta fiabilidad y baja } \\
\text { latencia } \\
\text { - Asignación flexible de } \\
\text { ancho de banda } \\
\text { - Puertas de enlace de red } \\
\text { privada } \\
\text { c Compatibilidad con la } \\
\text { corriente } \\
\text { Auriculares y dispositivos } \\
\text { - Software actualizable - } \\
\text { capaz de } \\
\text { Apoyando futuras mejoras } \\
\text { de producto } \\
\text { - Integración eficiente de } \\
\text { aplicaciones } \\
\text { - Ventajas de la tecnología } \\
\text { IP } \\
\text { - Diseñado para alojar cargas } \\
\text { útiles secundarias } \\
\text { - }\end{array}$ \\
\hline
\end{tabular}

Iridium también se puede utilizar para proporcionar un enlace de datos a otros satélites en el espacio, lo que permite el mando y control de otros activos espaciales, independientemente de la posición de las estaciones terrestres y pasarelas.

La red Iridium esa una constelación de satélites de baja orbita en malla interconectada que se comunican con los otros satélites cercanos, hacia adelante, hacia atrás y en órbitas adyacentes ver 
figura 10. Por lo tanto, la red satelital, al igual que una red celular, gestiona las comunicaciones de voz y datos de forma automática desde un haz puntual a otro dentro de la pisada de los satélites y de un satélite al siguiente mientras pasan por el cielo.

La llamada se retransmite desde un satélite a otro en la constelación sin tocar suelo hasta que se realiza un enlace descendente en un Gateway Iridium y posteriormente es conectado en la Red Pública de Telefonía Conmutada (PSTN) para la transmisión a su destino (IEC-Telecom, 2015).

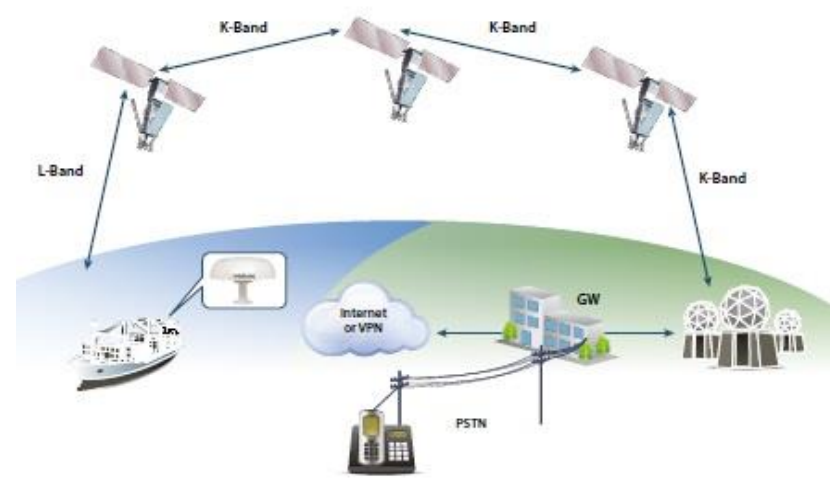

Figura 11. Enlace entra satélites, usuario y telepuerto.

Servicios

Telefonía

Un servicio de telefonía bidireccional basado en prioridades. Utilizado para operaciones rutinarias barco-tierra, tierra-tierra y comunicaciones de buque a buque

Enlace de datos

Un servicio de datos bidireccional basado en prioridades. Utilizado para operaciones rutinarias barco-tierra, tierra-tierra Comunicaciones

Emisión

Un nuevo enfoque de un solo sentido basado en servicio de datos. Se puede utilizar para la distribución de Información de seguridad (MSI)

Banda ancha

Un conjunto de datos por el servicio de banda ancha bidireccional, utilizado para comunicaciones rutinarias.

\section{El sistema marítimo de socorro y seguridad mundial "SMSSM"}

(Nautical, 2018) Es un sistema de seguridad internacional, que utiliza tecnología satelital y terrestre y sistemas de radio a bordo de buques para evitar accidentes y para alertar automáticamente a las autoridades de rescate y buques cercanos rápidamente en caso de emergencia. 
Según la convención de Seguridad de la Vida en el Mar (SOLAS), los buques de carga de 300GRT y más y todos los buques de pasajeros en viajes internacionales deben estar equipados con equipos de radio y satélite que cumplan con los estándares internacionales. Más información:

(Nautical, 2018) En Mayo del 20181 el Comité de Seguridad Marítima (MSC) de la Organización Marítima Internacional (OMI) acordó reconocer que la red Iridium cumple con todos los criterios de la OMI necesarios para proporcionar servicios satelitales móviles en el GMDSS y adoptar la "Declaración de reconocimiento" Propuesto por los Estados Unidos.

Este es un logro significativo después de décadas de monopolio de la industria satelital en el que solo una empresa estaba autorizada para proporcionar el servicio satelital GMDSS. Hasta ahora, Inmarsat era el único proveedor de servicios de comunicación satelital aprobados por GMDSS. Sus servicios Inmarsat $\mathrm{C}$ y Fleet 77 han mantenido a 1,5 millones de marinos seguros en el mar todos los días desde el ínicio del GMDSS en 1999.

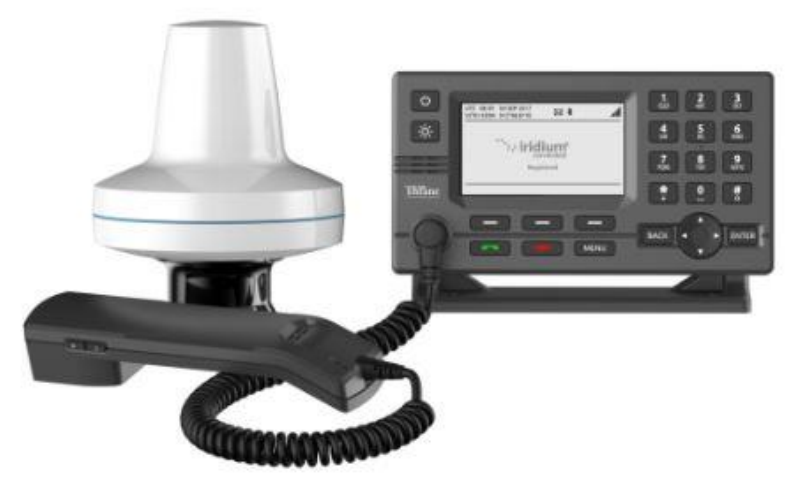

Figura 12: Terminal Iridium GMDSS LT3200

(www.iridium.com/services/gmdss/, 2018) La terminal LT-3200 se utilizar para el servicio Iridium GMDSS, fabricado por Lars Thrane A / S. El LT-3200 ofrecerá servicios comerciales y de seguridad, todo en un terminal especialmente diseñado. La antena de bajo perfil es fácil de instalar y entregará vOz, LRIT, SSAS, MSI (Maritime Safety Information) y alerta. Tiene capacidad de conectarse una impresora los cual lo equipara al Inm-C a través de la terminal de bajo costo.

Según Iridium (Iridium.com), los operadores de buques a menudo llevan múltiples sistemas de comunicaciones con el fin de cumplir con los requisitos del SMSSM exigidos por SOLAS y luego satisfacer las necesidades de comunicaciones operacionales del buque. Si su aplicación tiene éxito, Iridium dice que estará en condiciones de ofrecer terminales que entregan comunicaciones de seguridad y operacionales desde una sola caja. Para ello, la empresa ya se ha comprometido con fabricantes de hardware de comunicaciones marítimas establecidos para la producción y certificación de terminales SMSSM que funcionan con la red Iridium.

La constelación satélite de baja órbita terrestre de Iridium proporciona una cobertura global incluidas las regiones polares, Figura 12, que no están cubiertas efectivamente por las redes de satélites de inmarsat, brindando servicio de comunicaciones y seguridad a las nuevas rutas polares, y 
donde se prevé que la demanda de comunicaciones de voz y datos sea más elevada Y las rutas comerciales se aventuran en latitudes cada vez más altas.
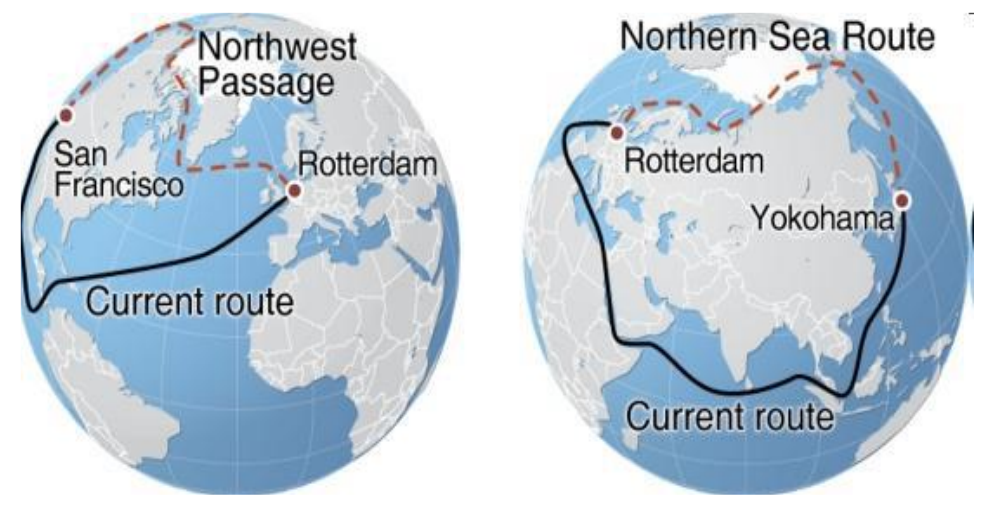

Figura 13. Iridium es el único proveedor de MSS que cubre GMDSS Región A4, servicio sólo por radio $\mathrm{HF}$.

Además, Iridium espera que sus terminales del SMSSM tengan una longevidad operacional de casi 20 años, eliminando la necesidad de que los dueños y operadores de embarcaciones compren nuevos equipos cada pocos años.

\section{Thuraya}

(IEC-Telecom, 2015) Thuraya es un proveedor regional de telefonía satelital, con servicio en CEE / CIS, WCARO, ESARO (excluyendo Sudáfrica, Lesotho, Swazilandia;

La compañía es competidor de Inmarsat en el servicio satelital móvil. Tiene su sede en los Emiratos Árabes Unidos y distribuye sus productos y servicios a través de proveedores de servicios autorizados. Mientras el usuario esté dentro del área de cobertura, Thuraya ofrece conectividad satelital, que incluye voz, datos (9.6kbps a 444kbps), fax, SMS y GPS.

\section{Cobertura}

(IEC-Telecom, 2015) Thuraya opera dos satélites geoestacionarios en su segmento espacial. Fueron lanzados en junio de 2003 (Thuraya-2, $25^{\circ}$ Este) y enero de 2008 (Thuraya-3, $154^{\circ}$ Oeste). Su órbita geosíncrona los hace aparecer en un punto fijo sobre la tierra desde la perspectiva de los usuarios. Debido a que Thuraya no tiene un satélite en las Américas. 


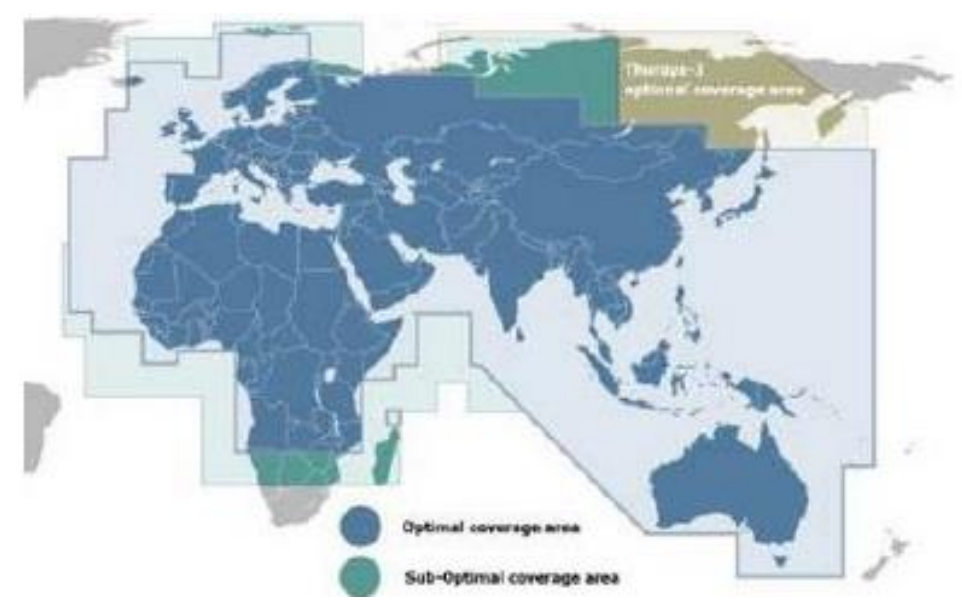

Figura 14: Cobertura de Thuraya.

\section{Segmento espacial}

El programa Thuraya consta de dos satélites OSG estabilizado entre tres ejes y equipados con múltiples cargas útiles de haz puntual de gran potencia para servicios móviles. Hay un satélite en órbita a $44^{\circ} \mathrm{E}$ y uno de repuesto en tierra. El segundo satélite se lanzará después del primero para dar más capacidad y como reserva.

El satélite transmite y recibe llamadas a través de una antena única de 40 pies (aproximadamente 12 m) de apertura y utiliza 250-300 haces puntuales para servicios telefónicos móviles compatibles con el sistema GSM.

El procesamiento de la señal digital a bordo del satélite en camina las llamadas desde una unidad de mano a otra o a la red terrenal. El sistema de satélite tiene hasta 13750 canales dúplex simultáneos para los enlaces de comunicación siguientes: enlace estación de cabecera-móvil, enlace móvilestación de cabecera y enlace móvil-móvil.

El sistema puede aceptar cambios en el tráfico del Thuraya por medio de una carga útil reprogramable en el satélite. Con esto se pueden introducir modificaciones en la zona de cobertura del satélite después del lanzamiento y actualizaciones las características en zonas geográficas en que haya una elevada demanda de tráfico. El procesador crea un gran número de haces puntuales que se reorientan hacia donde sea necesario, incluso orientar el satélite en órbita: desde Grandes ciudades o zonas rurales o barcos en el mar.

\section{Red}

La red Thuraya es muy similar a otras redes de satélite MSS o convencionales. Un satélite GEO, que constituye el segmento espacial, es operado y administrado por una red terrestre conocida como "segmento terrestre" (equivalente al LES en terminología VSAT o SAS por Inmarsat). El segmento terrestre incluye el Centro de operaciones satelitales (en Sharjah, EAU), que monitorea y controla el movimiento de los satélites, asegurando el mantenimiento general y continuo de los satélites en órbita geo-síncrona. El segmento de usuario comprende los terminales de usuario que permiten a los suscriptores interactuar con el sistema satelital y obtener acceso a la red: 


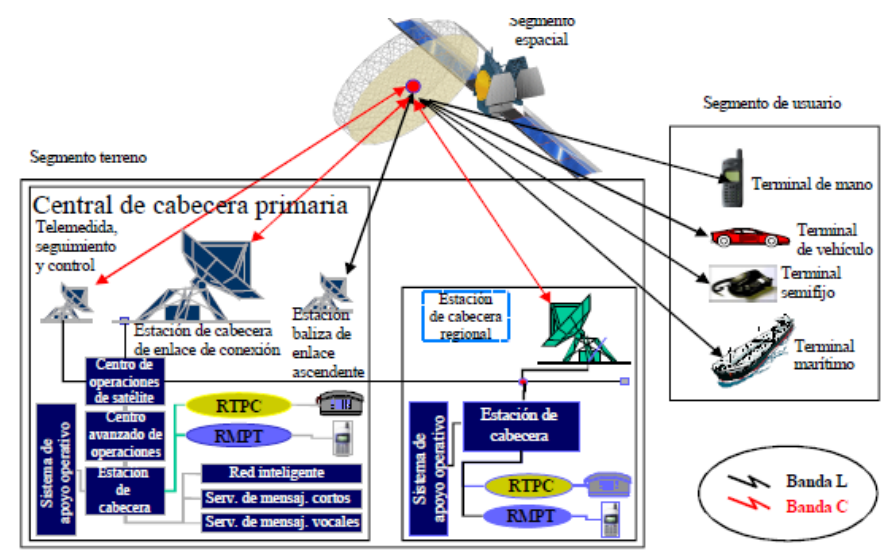

Figura 14: Red de Thuraya.

El segmento terrestre está constituido por una estación de cabecera primaria situada en los Emiratos Árabes Unidos que lleva el control general de toda la Red Thuraya. La estación de cabecera primaria consta de los elementos siguientes:

El centro avanzado de operaciones que lleva la gestión central de los recursos compartidos de satélite y configuración en consecuencia la carga útil de este.

Las estaciones de cabecera que dan acceso a la RTPC para el origen y la terminación de las Llamadas con los usuarios móviles y que da acceso a la red móvil terrestre pública (RMTP) y a la RDCP a través de la RTPC. También se establece la conexión directa de la señalización y de los enlaces troncales con la RMTP.

El sistema de apoyo operativo (OSS) tiene las funciones de atención y facturación centralizada al cliente y la personalización de tarjetas y gestión centralizada del sistema de conmutación de red (NSS).

Los operadores regionales de la estación de cabecera pueden ser propietarios de las centrales de cabeceras regionales y explotarlas. La interfaz con otras centrales de cabecera Thuraya se conectará a través del satélite y de las redes terrenales públicas fija y móvil GSM.

(thuraya, 2018) Con acuerdos de roaming establecidos en 161 países, puede proporcionar servicios de roaming confiables para para clientes de pospago y prepago en más de 395 redes GSM en todo el mundo. Los suscriptores GSM de esas redes también pueden insertar su SIM GSM en un teléfono Thuraya para disfrutar de los servicios de roaming en la red satelital de Thuraya, para que puedan permanecer conectados cuando no tienen cobertura GSM, o cuando viajan, los cargos de roaming terrestre son elevados.

El espectro de frecuencia es en banda $L$ en las Regiones 1 y 3 y se le ha asignado espectro en la banda 1525-1559 MHz/1626,5-1660,5 MHz, en la primera reunión de revisión de operadores (ORM) del SMS/OSG en banda L para las Regiones 1 y 3. Este proceso de coordinación es un proceso multilateral que se ajusta a la reglamentación de la UIT y como tal tiene el reconocimiento de ésta participando en él todos los operadores de satélite OSG y de redes. 
(UIT-SMS, 2010)

Enlaces de usuario $1525-1559 \mathrm{MHz}$ (espacio-Tierra)

1626,5-1660,5 MHz (Tierra-espacio)

Enlaces de conexión $3400-3625 \mathrm{MHz}$ (espacio-Tierra)

6425-6725 MHz (Tierra-espacio)

Thuraya MarineComms brinda a los mercantes, pesca, gobierno, marina, guardacostas y usuarios de ocio conectividad a precios competitivos, robustos y confiables, que abarcan datos de voz, banda estrecha y banda ancha. La red satelital de banda L puede asignar capacidad donde y cuando sea necesario, proporcionando un servicio que cubre algunos de los puertos y rutas de navegación más concurridas del mundo.

El servicio M2M marítimo de Thuraya le permite enviar actualizaciones a su flota de manera rentable. La red de baja latencia garantiza que la información crítica se transmita en tiempo real para permitirle tomar decisiones en el acto.

Thuraya Maritime M2M permite la captación de datos más inteligente, más racionalizada y automatizada a través de redes satelitales y GSM. Facilita el envío de datos procesables directamente al operador o gerente, mejorando así el rendimiento de los activos y la eficiencia del proceso (thuraya, 2018).

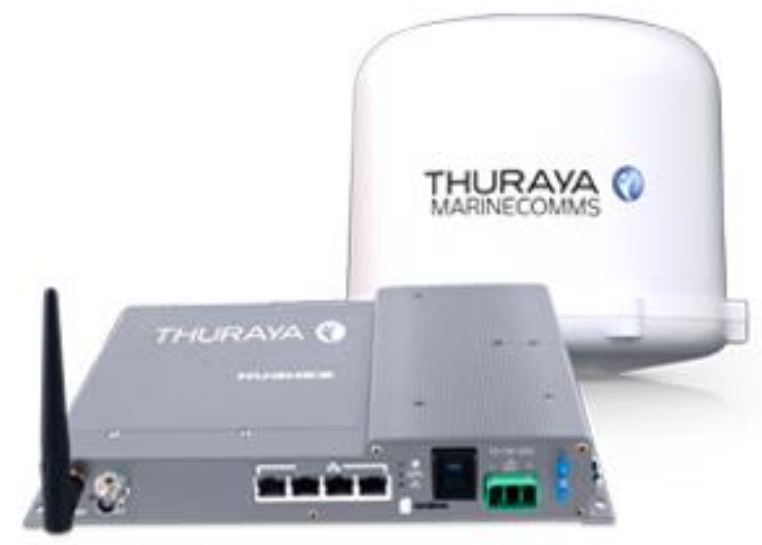

Figura 15: Equipo Marino Thuraya Atlas +IP

Las prestaciones del servicio marino son:

- Voz analógica y Voip.

- Data a Standard IP bajada/subida: 444/404 kbp.

- Streaming IP subida/bajada: 16kbps, 32kbps, 64kbps, 128kbps; 256kbps or 384kbps (Asimétrico).

- Simultaneous voice and data via built-in VoIP ATA. (thuraya, 2018) 


\section{VSAT Marítimo (MVSAT)}

La tecnología VSAT se utiliza para comunicaciones satelitales bidireccionales para Internet, datos y telefonía, generalmente en áreas rurales y entornos hostiles, en nuestro caso, en entornos marítimos.

Normalmente, las soluciones VSAT en el mercado marítimo se entregan como un paquete, que incluye el segmento espacial satelital, equipos y servicios de telefonía e internet.

Los servicios VSAT históricamente han operado en la banda $\mathrm{Ku}$ y en la banda $\mathrm{C}$ para el mercado comercial, utilizando satélites ubicados en la ubicación orbital GEO.

Debido a la ubicación de los satélites en combinación con el movimiento de los buques, se requieren antenas estabilizadas (X, Y, Z, AZ y EL) con seguimiento por medio de GPS. Una antena VSAT marítima estabilizada generalmente tiene una antena circular (a menudo oculta dentro de una cúpula) de 2,4 metros o menos de diámetro. Las velocidades de datos suelen oscilar entre 64 Kbps y 8 Mbps, pero están disponibles velocidades de datos más bajas y mucho más altas figura 17.

Una antena y un transceptor que se colocan al aire libre (generalmente dentro de una cúpula); en el área marítima, esto puede denominarse "Unidad de cubierta superior - ADU".

Una unidad interior que interactúa con la unidad exterior y controla la antena. En el sector marítimo, esto puede denominarse "Unidad de cubierta inferior - BDU" ver figura 16. (Marlink.com, 2019).

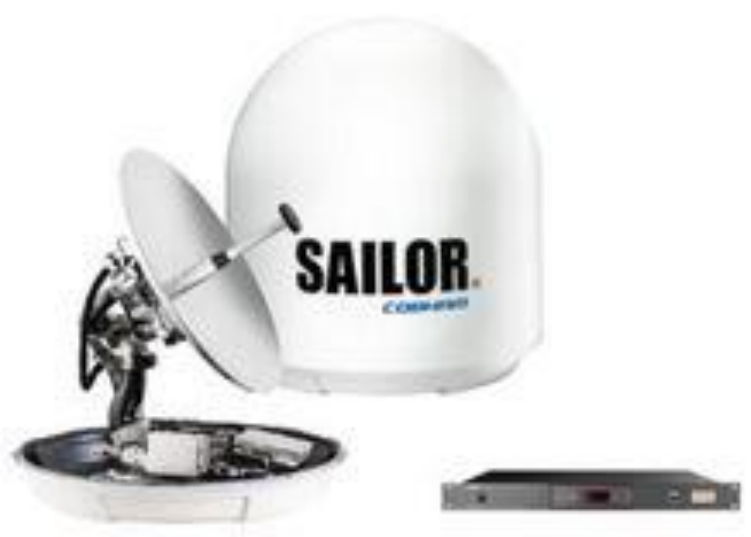

Figura 16: VSAT Marítimo con BDU y ADU. 


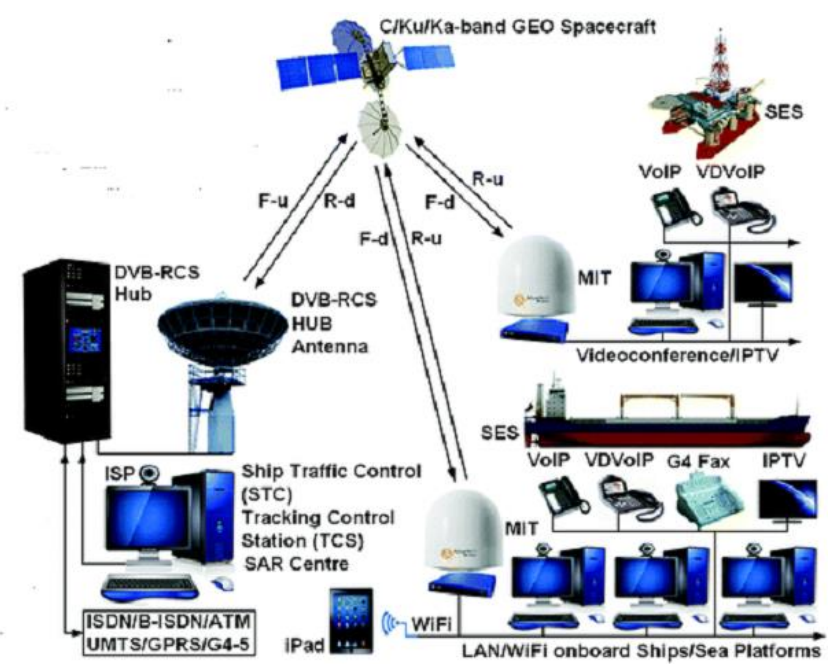

Figura 17: Red de datos de un MVSAT

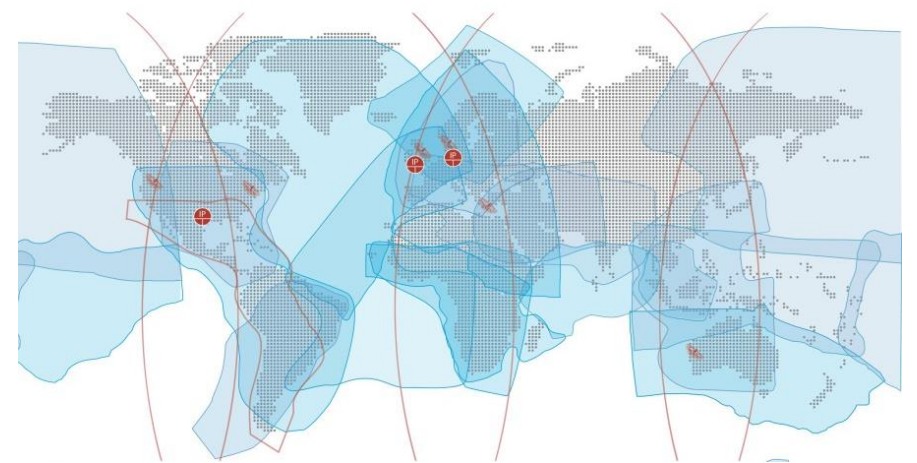

Figura 18: Cobertura de VSAT

\section{Conclusiones:}

- Los operadores están ofreciendo mayor ancho de banda para satisfacer la creciente demanda de Redes de datos para aplicaciones de seguridad, navegación, Block chain y Smart Ships.

- El aporte a la industria marítimas, en dar un servicio confiable, seguro y eficiente a la logística del transporte de carga al facilitar las comunicaciones entre cliente, buque y puerto.

- Ha sido la tecnología que empujo a GMDSS dando unas comunicaciones confiables, sin problemas de atmosféricos y conectando en tiempo real buque con el centro de búsqueda y rescate,

- El servicio de comunicaciones satelitales terrestre se ha visto mermada por la tecnología de comunicaciones de fibra óptica de última generación, pero el mercado de las comunicaciones marítimas está creciendo y se espera un manejo de ancho de banda de miles de Terabytes por año.

- Las comunicaciones de voz paso a segundo nivel en demanda, hoy por hoy son las comunicaciones de datos,

- La oferta de equipos que cumplan con los requerimientos de GMDSS por Iridium en los 
próximos años reducirá los servicios de Autoridades contables de Radio y activación de terminales, porque no lo requerirá.

\section{Bibliografía}

Handbook, I. (2004). Inmarsat Maaritime Communication HandBook. En Inmarsat. London: Inmarsat Customer Services.

IEC-Telecom. (11 de 6 de 2015). MSS-Telecoms-Handbook-v3.0-. (I. Telecom, Ed.) Obtenido de www.iec-telecom.com: http://www.safa-telecom.com/wp-content/uploads/2016/06/MSSTelecoms-Handbook-v3.0-Chapter-4-MSS-IEC-Telecom-2015.pdf

Inmarsat/maritime-safety. (29 de Mayo de 2018). https://www.inmarsat.com/. Obtenido de maritimesafety: https://www.inmarsat.com/service/maritime-safety/

Latamsatelital, R. d. (29 de Junio de 2019). wmw.latamsatelital.com. Recuperado el 23 de Julio de 2019, de https://latamsatelital.com/inmarsat-amplia-global-xpress-artico/

Leppo, C. (12 de Marzo de 2019). What satellite communication technology is better VSAT Inmarsat or Iridium. Obtenido de https://www.quora.com/: https://www.quora.com/What-satellitecommunication-technology-is-better-VSAT-Inmarsat-or-Iridium

Marlink.com. (5 de Junio de 2019). ¿Qué es VSAT MARÍTIMO? Obtenido de https:/ / marlink.com: https://marlink.com/what-is-maritime-vsat/

Nautical. (29 de Mayo de 2018). bttps:// wmm.nautical.es/. Obtenido de iridium and inmarsat fleetbroadband gmdss services approved: https://www.nautical.es/iridium-and-inmarsatfleetbroadband-gmdss-services-approved-by-imo/?lang=en

Satelite, L. (2016 de Agosto de 2016). bttp:/ / latamsatelital.com/next-la-constelacion-nueva-generacioniridium/. (Latansatelital, Ed.) Recuperado el 2019, de http://latamsatelital.com/next-laconstelacion-nueva-generacion-iridium/: http://latamsatelital.com/

Symposium, J. C. (November 2013.). ) Iridium GMDSS Service Provider Recognition Process. Japan Coast Guard Symposium, (págs. 19-20).

technologies, S. (18 de Mayo de 2015). Skyware_Technologies_The_Basics_on_Imarsats_GX_network.pdf. Obtenido de http://www.skywaretechnologies.com/news/item/82-global-xpress-what-is-it: http://www.skywaretechnologies.com

thuraya. (12 de 7 de 2018). https://thuraya.com/marine-comms. Obtenido de https://thuraya.com/marine-comms: https://thuraya.com/

UIT-SMS. (2010). Servicos Moviles por Satelite. En UIT-SMS, Servicos Moviles por Satelite (págs. 125130). London: UIT.

UPV. (https://www.uv.es/ hertz/hertz/Docencia/teoria/Historia.pdf). bttps://mmw.uv.es/ hertr/ hertr/Docencia/teoria/Historia.pdf. Recuperado el 22 de 7 de 2019, de https://www.uv.es/ hertz/hertz/Docencia/teoria/Historia.pdf

Wikipedia. (2019). https:/ / es.wikipedia.org/wiki/Inmarsat. (Wikipedia) Recuperado el 7 de Julio de 2019, de https://es.wikipedia.org/wiki/Inmarsat

www.iridium.com/services/gmdss/. (29 de Mayo de 2018). https:// wmw.iridium.com/services/gmdss/ . Obtenido de https://www.iridium.com/: https://www.iridium.com/services/gmdss/ 